Cita bibliográfica: Jaramillo Escobedo J. V., Luyando Cuevas, J. R., Guzowski, C. y Zabaloy, M. F. (2019). Eficiencia energética y sustentabilidad en hoteles de sol y playa en el Noreste de México. Investigaciones Turísticas (18), pp. 42-70. http://dx.doi.org/10.14198/INTURI2019.18.03

\title{
Eficiencia energética y sustentabilidad en hoteles de sol y playa en el Noreste de México
}

\author{
Energy efficiency and sustainability in sun and beach hotels in Northeast Mexico
}

José Vicente Jaramillo Escobedo iD, Universidad Autónoma de Nuevo León, México jaramillo vicente@hotmail.com

José Raúl Luyando Cuevas (iD, Universidad Autónoma de Nuevo León, México irlc9@hotmail.com

Carina Guzowski, Universidad Nacional del Sur, Argentina

cguzow@criba.edu.ar

María Florencia Zabaloy, Universidad Nacional del Sur, Argentina

florencia.zabaloy@uns.edu.ar

\section{RESUMEN}

El objetivo del presente trabajo es evaluar si la oferta turística en hoteles de los municipios conurbados de Tampico, Madero y Altamira en el estado de Tamaulipas en México, contempla medidas de eficiencia energética, en el sentido de procurar una estancia turística que gradualmente pueda ser más sustentable en la zona. Para lograrlo, se realizaron encuestas a una muestra representativa de hoteles afiliados a la Asociación de Hoteles del Sur de Tamaulipas A.C., encontrándose que pocos de estos hoteles están llevando a cabo acciones sustanciales para reducir el consumo de energía mediante prácticas internacionales de eficiencia energética y, por tanto, existe la necesidad de implementar en la zona estudiada políticas que apoyen o estimulen este tipo de prácticas.

Palabras clave: Consumo de energía, Gases de Efecto Invernadero, Turismo, Playa Miramar, Tamaulipas.

\section{ABSTRACT}

The objective of this paper is to assess whether the tourist offer in hotels in the municipalities surrounding Tampico, Madero and Altamira, in the state of Tamaulipas in Mexico, contemplates energy efficiency measures so as to gradually ensure more sustainable tourist stays in the area. To achieve this, surveys were conducted among a representative sample of the hotels 
affiliated to the Association of Hotels of the South of Tamaulipas A.C. The findings reveal that few of these hotels are carrying out substantial actions to reduce energy consumption through international practices of energy efficiency and, therefore, there is an urgent need to implement policies that support or stimulate these types of practices in the area studied.

Keywords: Energy consumption, Greenhouse Gases, Tourism, Miramar Beach, Tamaulipas.

\section{INTRODUCCIÓN}

El turismo de sol y playa es el pilar de este sector a nivel mundial, en gran medida por el número de turistas que atrae y los ingresos que genera. La tasa de crecimiento del total de la actividad turística en el mundo ha sido positiva, con respecto tanto a llegada de turistas como a ingresos ${ }^{1}$. De acuerdo a estadísticas presentadas en el barómetro de la Organización Mundial de Turismo [OMT] $(2017 ; 2019 a)$, la actividad turística tuvo una tasa de crecimiento media anual del 5\% entre los años 2010 y 2018, la cual cabe señalar, se sostuvo incluso durante la crisis económica mundial entre los años 2008 y 2009. En este mismo sentido la OMT (2019a), también menciona que, para el 2019, se espera un crecimiento del $3 \%$ al $4 \%$ con respecto al año anterior, lo cual manifiesta una propensión creciente en el sector. Un dato relevante que ayuda a entender esa tendencia positiva en el tiempo, es la evolución que ha experimentado el sector desde el año 1950 al 2018 por concepto de llegada de turistas, la cual, se observa en la gráfica 1.

Gráfica 1.- Llegada de turistas a nivel mundial

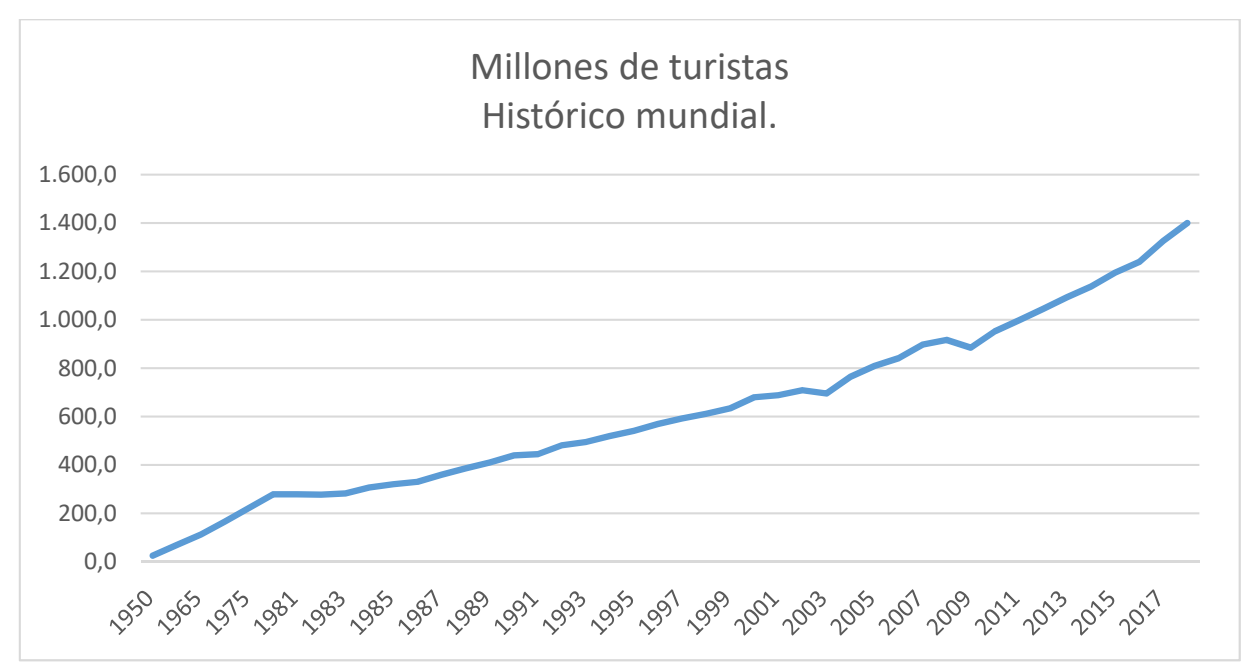

Fuente: Elaboración propia con datos estadísticos de la Organización Mundial del Turismo (Barómetro OMT -Volumen 15, agosto de 2017-Anexo Estadístico). Años actualizados para turistas e ingresos 2005, 2010, 2014, 2015, 2016, 2017 y 2018.

1. Tan solo en el 2016 de acuerdo a la Organización Mundial del Turismo, el ingreso por turismo internacional superó el billón de dólares (un billón doscientos veinte mil millones de dólares) y en el rubro de llegadas internacionales los 1235 millones de turistas; para el 2017 se incrementó el ingreso hasta alcanzar un billón trescientos treinta y dos mil millones de dólares y las llegadas a 1326 millones de turistas. (OMT, 2018b). En 2018 se alcanzó una cifra en el ingreso por un billón setecientos mil millones de dólares y un flujo de visitantes cercano a los 1400 millones de turistas (OMT, 2019b). 
Además, cabe señalar que de acuerdo al dato más reciente de la OMT, en el año 2018 esta actividad representó el $10 \%$ del Producto Interno Bruto (PIB) a nivel mundial y el mismo porcentaje en el empleo, es decir, uno de cada diez puestos de trabajo a nivel mundial son creados por la industria turística (OMT, 2018c).

Para el caso de México, el turismo es una de sus principales fuentes de ingresos por concepto de divisas y generador de empleo, pues es considerado uno de los principales destinos a nivel mundial, ocupando actualmente el sexto lugar en cuanto a la recepción de turistas y décimo cuarto por concepto de entrada de divisas (Paredes, 2018).

En México y el mundo, el destino turístico que convoca playa Miramar para los municipios de Ciudad Madero, Tampico y en menor medida Altamira ${ }^{2}$ no ha tenido la relevancia de destinos como Cancún y Playa del Carmen en Quintana Roo ${ }^{3}$, Los Cabos en Baja California Sur, Puerto Vallarta en Jalisco y Huatulco en Oaxaca. Pues a pesar de tener este atractivo natural, esta región se significó más por tener una refinería en Ciudad Madero, un puerto y la producción de resinas derivadas del petróleo en Altamira y el comercio en Tampico 4 . La playa quedó por largo tiempo en segundo término y no fue sino hasta la crisis en el sector petrolero -cuando se redujo significativamente la refinación de petróleo o quedó por lapsos inoperante la planta de refinación- que las autoridades estatales y municipales voltearon a ver este atractivo como una salida a la situación económica y social que se había generado. Por mucho tiempo, la playa se consolidó solo como un destino regional, es decir, captaba turistas de estados vecinos, principalmente del estado de Nuevo León, generalmente turistas de medianos y bajos recursos que acudían en periodos determinados -esencialmente en semana santa y los meses de julio y agosto-, es decir, no se le apreciaba en su esencia como un destino de importancia turística en el plano nacional e internacional. En el primer caso, entre otras cosas, se tenía la percepción de ser una playa contaminada al encontrarse junto a una refinería y en el segundo, por ser un destino turístico prácticamente desconocido. Pero ante el nuevo escenario, los gobiernos estatales y municipales se han dado a la tarea de mejorar las condiciones del recurso natural y su entorno, con el fin de consolidarlo como un destino turístico reconocido en los dos ámbitos antes señalados. Por ejemplo, en el plan municipal de desarrollo de Ciudad Madero 2013-2016 (Gobierno Municipal de Ciudad Madero, 2013, p. 15), en el punto 7, referente al plano turístico, se señala la acción siguiente: "La limpieza permanente, la certificación de la playa, la forestación de la misma y la regulación de la zona federal formarán parte de nuestros compromisos fundamentales en este sector" y en el punto 9, referente a la sustentabilidad, hacen el compromiso siguiente: "Se incorporarán, campañas permanentes de concientización para todos los adultos y niños sobre la importancia de la ecología y el concepto de sustentabilidad". Cabe

2. Los municipios de Altamira, Ciudad Madero y Tampico conforman la denominada Zona Conurbada del Sur de Tamaulipas.

3. Son las playas, por mucho, más reconocidas a nivel mundial y nacional.

4. Un informe realizado por el Programa de Naciones Unidas para el Medio Ambiente (PNUMA), La Secretaría de Medio Ambiente y Recursos Naturales (SEMARNAT) y el gobierno de Tamaulipas, describe a los municipios de acuerdo a la preponderancia de sus actividades, destacando en Altamira las actividades pesqueras, agropecuarias e industriales; en Ciudad Madero las actividades encaminadas a la explotación de hidrocarburos, de petroquímica básica y secundaria; mientras que en Tampico las actividades enfocadas al comercio y los servicios turísticos son las más importantes (Programa de Naciones Unidas para el Medio Ambiente (PNUMA), Secretaría de Medio Ambiente y Recursos Naturales (SEMARNAT) y Gobierno de Tamaulipas, 2015). 
también señalar que la Secretaría del Medio Ambiente y Recursos Naturales (SEMARNAT), en su programa de playas limpias, considera a playa Miramar desde el 2013 hasta junio del 2019 como una playa apta, en lo referente a la calidad bacteriológica del agua ${ }^{5}$, ubicándola según datos oficiales en 2018, entre las 10 playas más limpias de México de acuerdo a la Norma Mexicana NMX-AA-120-SCFI-2016 (SEMARNAT, 2017).

Ahora que el turismo ha tomado preponderancia como actividad económica en Tamaulipas, cabe indicar que el municipio de Ciudad Madero es el que más turistas atrae. En la gráfica 2, se puede apreciar que desde el año 2010 existe un repunte en el porcentaje de ocupación hotelera en la entidad, pues pasó de $28.93 \%$ a $55.96 \%$ en 2014 . Después de este último año decae para 2015 y 2016, pero aún para este último se mantiene por arriba del 50\%. Cabe señalar que el porcentaje repunta en 2017, al tener el estado una ocupación hotelera del $53 \%$, impulsada principalmente por la afluencia turística al municipio de Ciudad Madero ${ }^{6}$ que participó para este año con el $49 \%$ del total estatal (Macías, 2017). Otra situación que se debe indicar es que aún con el repunte no se ha alcanzado la media nacional la cual era en el 2016 de 56.17\% (Secretaría de Turismo [SECTUR], 2018).

Gráfica 2.- Ocupación hotelera en Tamaulipas

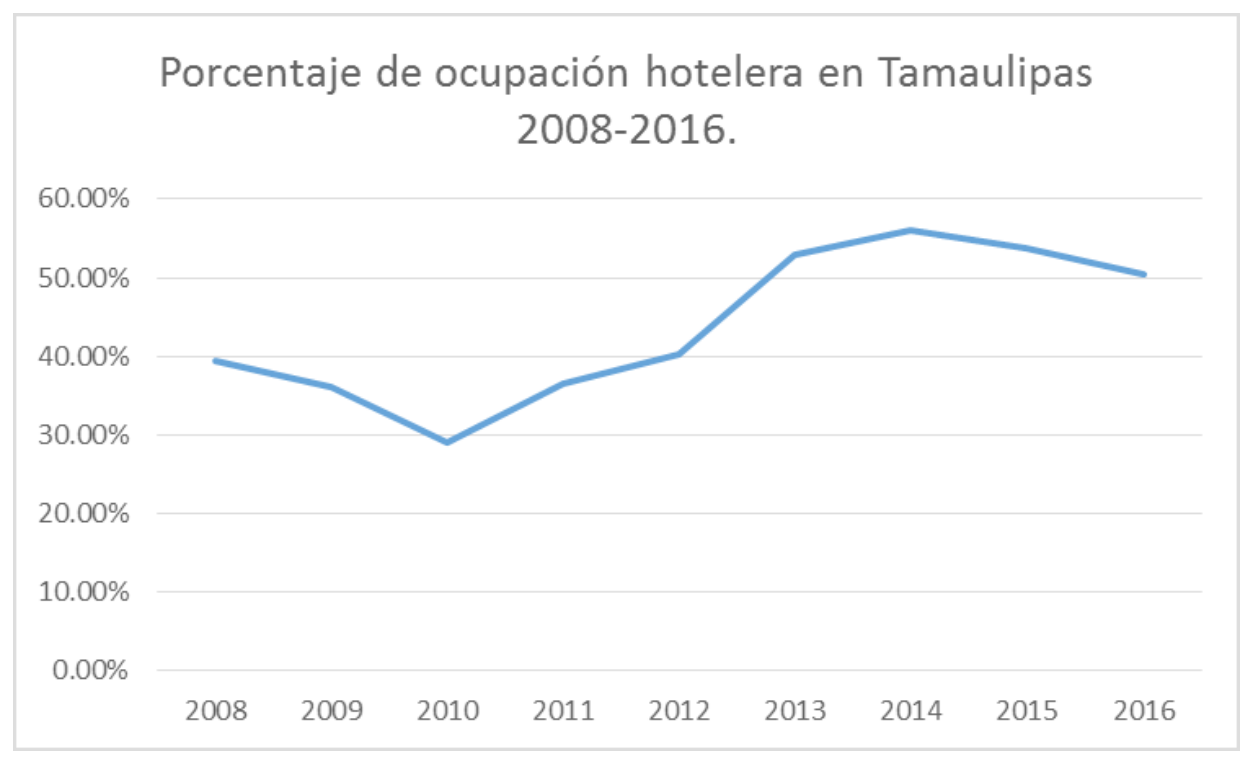

Fuente: Elaboración propia con datos estadísticos de la Secretaria de Turismo. Información Turística por Entidad Federativa. (2018).

Sin embargo, también se deben considerar los efectos negativos que puede tener esta actividad para el desarrollo sustentable de la región. En un principio la industria turística fue considerada una industria sin chimeneas (Dachary y Arnaiz, 2006), por tener la característica de no requerir de procesos de tipo industrial que son percibidos por la comunidad como contaminantes. Por lo tanto, el impacto ambiental de la actividad turística se ve reflejado

5. SEMARNAT (2019): Resultados de calidad de agua de mar. Ciudad Madero, Tamaulipas.

6. La playa Miramar, localizada en el municipio de Ciudad Madero, en los últimos años ha tenido un repunte importante en lo que respecta a llegada de turistas y ocupación hotelera (Excelsior, 2018). 
principalmente en el consumo de recursos tanto renovables como no renovables, consecuencia de la alta demanda de agua, electricidad, combustibles, entre otros, requeridos para desarrollar y sostener los destinos turísticos. Por ejemplo, en la gráfica 3 se pueden observar los consumos como parte de los impactos ambientales de las unidades económicas de alojamiento temporal, en lo referente a luz, agua y combustibles en comparación con el total de la economía en su conjunto. En ella se puede observar que el consumo de agua y luz son mayores con respecto al total de la actividad económica, sin embargo, hay que considerar que es una primera aproximación, pues no representan una visión integral de la problemática, puesto que no se están incluyendo los costos por externalidades negativas.

Gráfica 3.- Consumo de insumos en unidades económicas de alojamiento temporal

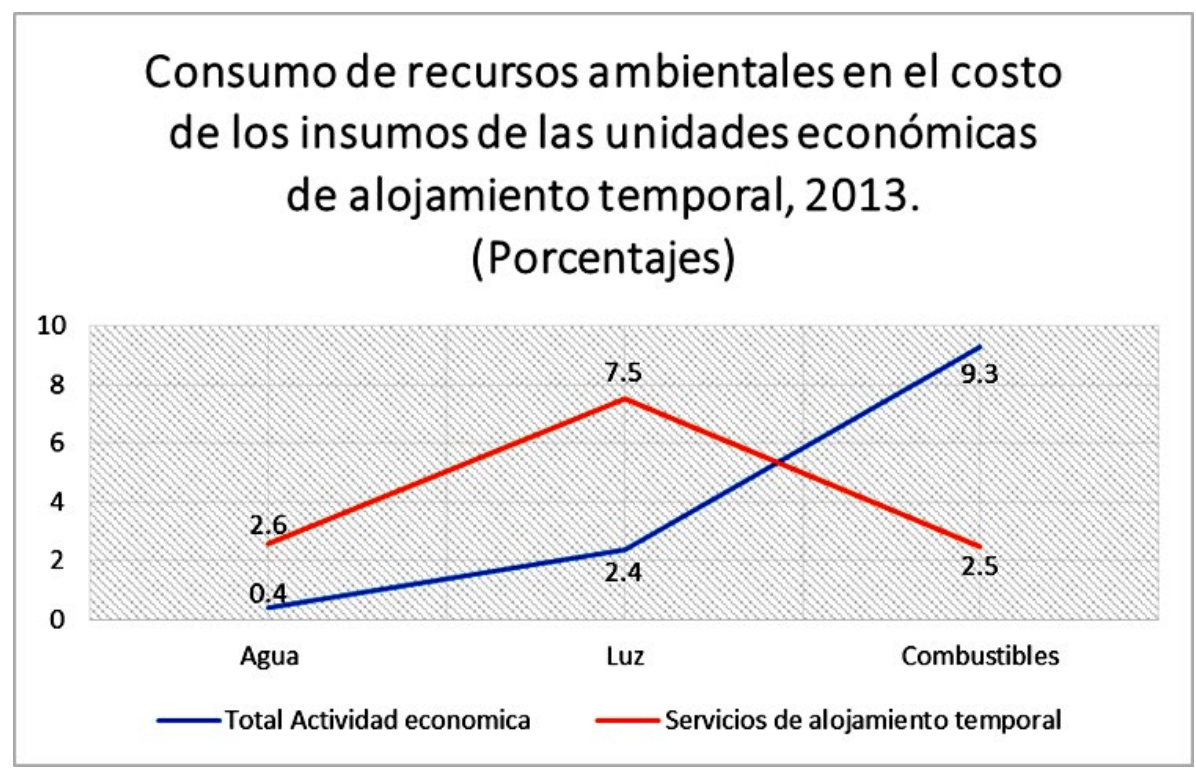

Fuente: Estadísticas a propósito del... día mundial del turismo (27 de Septiembre), Instituto Nacional de Estadística y Geografía (INEGI 2015).

En este sentido, una de las principales problemáticas que surgen es la relativa a los elevados niveles de consumo de energía eléctrica que realizan los visitantes en estos destinos, dado que la producción de este insumo es una de las principales fuentes de generación de dióxido de carbono $\left(\mathrm{CO}_{2}\right)^{7}$ y, por tanto, de los Gases de Efecto Invernadero a nivel mundial, existiendo una relación directamente proporcional entre mayor consumo de energía y emisión de $\mathrm{CO}_{2}$ (Wang, Lin y Luo, 2017, p. 1). En consecuencia la actividad turística se puede analizar desde dos vertientes. Por un lado, puede contribuir al crecimiento de las regiones donde se localiza el atractivo turístico, principalmente en la generación de ingreso y de empleo y, por otro, puede tener un impacto negativo en las condiciones sociales, económicas y ecológicas de sus habitantes, principalmente por la presión ejercida a los recursos naturales

7. Principalmente en países que como México aún se utiliza en mayor medida carbón, petróleo y gas para generar la energía eléctrica. De acuerdo al balance nacional de energía 2016 de la Secretaría de Energía (SENER), la producción de energía primaría tenía la siguiente estructura porcentual: Carbón 3.29\%, hidrocarburos 86.79\%, nucleoenergía $1.43 \%$ y renovables $8.49 \%$ (Secretaría de Energía [SENER], 2016, p. 23). 
(Idahosa, Marwa y Akotey, 2017). Por ejemplo, en el reporte elaborado por la Organización Meteorológica Mundial (OMM) y la Organización Mundial del Turismo (OMT), se menciona que para el 2005 el $4.9 \%$ del total de emisiones de $\mathrm{CO}_{2}$ a nivel global eran atribuidas a la actividad turística, destacando el transporte y alojamiento con el $75 \%$ y $21 \%$ respectivamente (OMT, OMM, 2008, p. 132). Esta contribución a las emisiones de $\mathrm{CO}_{2}$ desde el turismo ha crecido a lo largo del tiempo. En efecto, Lenzen et al. (2018) llevaron a cabo una investigación en la cual determinaron que esta contribución alcanzó hasta el $8 \%$ del total de emisiones de $\mathrm{CO}_{2}$ en el período de 2009 al 2013, tomando en cuenta consumos adicionales al transporte y alojamiento como lo son la alimentación y las compras durante las vacaciones por parte de los turistas, observando una relación positiva entre el incremento de riqueza (PIB per cápita) y la huella de carbono. Al respecto Olcina $(2012$, p. 16) menciona que: "Si consideramos el turismo como un país emisor de gases de efecto invernadero en su conjunto, ocuparía el $5^{\circ}$ lugar, por detrás de Estados Unidos, China, la Unión Europea y Rusia; de manera que estamos ante una actividad económica que resulta altamente impactante en relación con el control de emisiones de gases causantes del calentamiento planetario y que exige medidas urgentes para evitar que siga aumentando las emisiones a nivel mundial".

En este sentido, es importante señalar que la zona de estudio está caracterizada por una presión ambiental importante, relacionada a la producción de emisiones de $\mathrm{CO}_{2}$ provenientes del consumo de energía eléctrica. Este consumo es impulsado primordialmente por las actividades industriales y de servicios, destacando la participación de la industria petroquímica y otras actividades de prestación de servicios (entre las que se encuentra el turismo), las cuales son, junto al consumo residencial, las que demandan un alto consumo de energía $(97.69 \%)$ y generan más dióxido de carbono. En el cuadro 1 se muestra la emisión de $\mathrm{CO}_{2}$ a partir del consumo de la energía eléctrica en la zona de estudio.

Cuadro 1.- Estimación de la producción de dióxido de carbono $\left(\mathrm{CO}_{2}\right)$ por consumo de energía eléctrica en la zona de estudio.

\begin{tabular}{|l|c|c|}
\hline Tipo de Consumo & (MWh/mes) & $\mathrm{CO}_{2}$ (ton/mes) \\
\hline Doméstico & 48,348 & 21,998 \\
\hline Alumbrado Público & 2,616 & 1,190 \\
\hline $\begin{array}{l}\text { Industrial y de } \\
\text { servicios }\end{array}$ & 103,413 & 47,053 \\
\hline Bombeo de agua & 943 & 429 \\
\hline Agrícola & 32 & 15 \\
\hline Total & 155,352 & 70,685 \\
\hline
\end{tabular}

Fuente: IMEPLAN, basado en datos de CFE-INEGI y la Agencia Internacional de Energía. En PNUMA et al. (2015).

Del cuadro 1 se desprende que la zona de estudio requiere un consumo de energía que alcanza cifras anuales de alrededor de 1'240,956 mega watts hora (MWh) respecto a las actividades industriales y de servicios, destacando la generación de electricidad en el caso de Altamira, la petroquímica en Ciudad Madero y el sector servicios que se encuentra presente en los tres municipios y donde está incluida la actividad hotelera que, en su conjunto, llegan a producir el $66.57 \%$ de $\mathrm{CO}_{2}$ del total de las emisiones en la región. 
Ante el panorama descrito, el objetivo del presente trabajo es evaluar si la oferta turística en hoteles de los municipios conurbados de Tampico, Madero y Altamira en el estado de Tamaulipas en México, está contemplando medidas de eficiencia energética. Es decir, una contribución específica de la investigación es constatar el efecto que han tenido las políticas públicas sobre eficiencia energética en los hoteles del área turística que abarca la playa Miramar. En particular, las políticas dirigidas a reducir el consumo de energía o la utilización de fuentes de energías limpias -teniendo en cuenta que Tamaulipas es uno de los principales estados en México generadores de energía eólica- en estos establecimientos. Más aún, considerando que en el año 2017 el secretario de Desarrollo Urbano y Medio Ambiente del Gobierno de Tamaulipas señalaba que: "Se trabaja con firmeza en acciones rápidas y rentables para reducir las emisiones de carbono mediante una mayor eficiencia energética y aprovechamiento sostenible de la energía"(Cruz, 2017).

Ahora bien, considerando que la concentración masiva de turistas de sol y playa en destinos altamente turísticos como Cancún y Los Cabos y la problemática ambiental que ello conlleva, el gobierno mexicano propuso políticas encaminadas a fomentar la diversificación del desarrollo turístico, al señalar la necesidad de potencializar nuevos destinos de sol y playa, con el firme objetivo de que esto se haga desde una visión sustentable como se señala en el Plan Nacional de Desarrollo 2013-2018 (Gobierno de la República, 2013) y el Programa Sectorial de Turismo (SECTUR, 2013). Así mismo, la Organización para la Cooperación y el Desarrollo Económico (OECD, 2017), ha manifestado la importancia de la promoción de nuevos desarrollos turísticos, dado que "México se beneficiaría de dar un paso hacia un modelo de desarrollo turístico geográficamente distribuido" (p. 23). En este sentido, cabe destacar que existe escasa literatura y trabajos de investigación sobre eficiencia energética en la zona turística desarrollada a partir de la playa Miramar y, en general, en el estado de Tamaulipas a pesar de que fue uno de los estados en México que experimentó un alto incremento en su consumo de energía eléctrica (135\%) en proporción al incremento de su población e industria entre los años 1990 y 2006. Este incremento fue mayor al de Nuevo León (108\%) que en 2006 fue el segundo estado en el país que más energía eléctrica consumió debido a que es uno de los estados más industrializados en México, cuenta con mayor población y con un clima extremo ${ }^{8}$ (Comisión Federal de Electricidad [CFE], 2007). Por tanto, es imprescindible investigar las posibilidades de introducir sistemas de conservación de energía en Tamaulipas y primordialmente en el sector turístico considerando que se trata de una región (la zona de estudio ${ }^{9}$ ) a la que se le ha puesto especial interés en su desarrollo y principalmente a los hoteles que son el emblema de esta industria y su expresión más visible y concreta. Se estudiarán esencialmente políticas, reglamentos y programas relacionados con el diseño y los materiales utilizados en su construcción, el uso de técnicas y tecnologías que minimicen el uso de energía en todas las áreas del hotel, el uso de recursos renovables para el suministro de energía y la concientización en el uso de este recurso en gerentes, empleados y huéspedes, con el propósito último de entender

8. Consumo de energía eléctrica por entidad federativa (Gigawatts-hora). No se encuentran disponibles datos más recientes en la fuente del documento.

9. Tiene una temperatura promedio de $28.6^{\circ} \mathrm{C}$ entre los meses de abril a octubre que la hace propicia para el turismo de sol y playa durante 7 meses al año. 
y analizar desde una visión socio-ambiental, las condiciones que requieren estos nuevos destinos para tener una viabilidad sustentable.

En el apartado dos se describe la importancia de la eficiencia energética en el sector hotelero y la contribución que este último ha tenido en la generación de Gases de Efecto Invernadero (GEI). En el apartado tres se plantean los beneficios del uso de la energía de manera eficiente, así como los principales obstáculos para lograrlo, identificando entre otros, el factor económico en adquisición de equipamiento dado que representan un alto costo. En el apartado cuatro se hace referencia al proceso metodológico utilizado para recabar la información desde fuentes primarias mediante la técnica de encuesta describiendo el proceso realizado para terminar con el planteamiento de los resultados, conclusiones y recomendaciones que contribuyan al desarrollo turístico de la zona de estudio.

\section{EL RETO DE LA EFICIENCIA ENERGÉTICA EN EL MUNDO Y EN EL SECTOR HOTELERO}

“A medida que el mundo lucha por crear la cartera de energía adecuada para el futuro, la política de eficiencia energética está desempeñando un papel cada vez más importante. En los últimos años, la cantidad de dinero público y privado que se dedica a promover la eficiencia energética ha aumentado mucho"10 (Tietenberg y Lewis, 2012, p. 169). Además, de acuerdo a la Agencia Internacional de Energía, la eficiencia energética "Tiene el potencial excepcional de contribuir a largo plazo simultáneamente a la seguridad energética, al crecimiento económico, e incluso a una mejora de la salud y el bienestar; en particular constituye un medio clave para reducir las emisiones de GEI" (Agencia Internacional de Energía [AIE], 2015, p. 4). En este sentido, uno de los retos a los que se enfrenta la industria turística y en especial la hotelera en los destinos turísticos de sol y playa, es la reducción del alto consumo de energía requerido para ofrecer servicios a los huéspedes durante su estancia, principalmente en la climatización artificial para acondicionamiento de espacios. Esta problemática del uso intensivo de energía, trae consigo efectos negativos, no solamente en el alto costo de operación del establecimiento, sino también en la emisión de GEI que afectan a la población en general. Por tanto, la eficiencia energética se torna un elemento esencial en los esfuerzos que llevan a cabo los hoteles para reducir el alto consumo, ya sea mediante un cambio tecnológico en el equipamiento o induciendo prácticas sustentables en su operación. Oluseyi, Babatunde y Babatunde (2016) mencionan que se puede reducir los GEI mediante la implementación de medidas de eficiencia energética y la producción de energías renovables para el suministro. Por su parte, Prasad y Singh (2015) indican que a través de la eficiencia energética se pueden reducir las emisiones de $\mathrm{CO}_{2}$ y los costos de utilizar energía en los hoteles empleando energías renovables. Olcina (2012) señala que aquellos destinos turísticos que busquen mantener su competitividad -donde el servicio

10. Traducción propia: As the world grapples with creating the right energy portfolio for the future, energy efficiency policy is playing an increasingly prominent role. In recent years the amount of both private and public money being dedicated to promoting energy efficiency has increased a great deal. 
de alojamiento temporal juega un papel importante- deben adaptarse ante los nuevos escenarios relacionados con el cambio climático y que dentro de esta adaptación se pueden contemplar iniciativas como: a) Las buenas prácticas en hoteles, como sería el uso de energías alternativas y b) Los impuestos a las emisiones de GEl. Concluyendo de esta forma que "Mediante la reducción de las emisiones de $\mathrm{CO}_{2}$, los hoteles pueden hacer una contribución positiva al medio ambiente y, al mismo tiempo, reducir sus costes de operación" (Olcina, 2012, p. 26).

De acuerdo a la revisión de literatura, existen factores y áreas dentro de la estructura, distribución y operación de un hotel que determinan su consumo de energía -siendo la energía eléctrica la más utilizada, además del vapor, gas licuado, gas natural, gasolina y diésel- (Rotimi, Bahadori-Jahromi, Mylona, Godfrey y Cook, 2017; Sheng, Miao, Zhang Lin y $\mathrm{Ma}, 2018)$. Dentro de los factores, se pueden mencionar la temperatura exterior, la iluminación, la tasa de ocupación, el acondicionamiento de espacios mediante clima artificial por calefacción o aire acondicionado, características físicas del hotel, edad del edificio, cocción de alimentos, producción de agua caliente, uso de electrodomésticos, ascensores y escaleras eléctricas (Bohdanowicz, Churie-Kallhauge y Martinac, 2001; Bianco, Righi, Scarpa y Tagliafico, 2017; Idahosa et al., 2017; Oluseyi et al., 2016; Wang et al., 2017; Rotimi et al., 2017; Prasad y Singh, 2015; Tsoutsos, Tournaki, Frangou y Tsitoura, 2018). Existen distintas formas de estudiar las áreas que se deben tomar en cuenta para identificar el consumo energético de un hotel. Autores como Pieri, Tzouvadakis y Santamouris $(2015$, p.6) realizan una categorización que incluye 6 áreas: Áreas comunes, habitaciones, área de lavandería y limpieza, área de servicio de mantenimiento, área de comedor y área auxiliar y ocio. Por su parte autores como Tsoutsos et al. (2018, p.14), Bianco et al. $(2017$, p.3) y Bohdanowicz et al. $(2001$, p. 5$)$ realizan un balance en el consumo de energía eléctrica de hoteles, encontrando que la climatización demanda entre $50 \%$ y $62.51 \%$, los restaurantes, cafés y bares del $15 \%$ al $25 \%$, la producción de agua caliente del $9 \%$ al 30\%, la iluminación del $7 \%$ al $10 \%$ y el consumo eléctrico en habitaciones y otras áreas de servicio entre el $26 \%$ y $30 \%$. En este sentido autores como Molina, Borroto, Monteagudo, Velarde y Santiesteban (2017) mencionan que para la climatización de hoteles en regiones tropicales, se emplea hasta el $60 \%$ de la energía eléctrica del total utilizada para la operación de estos establecimientos.

En México, de acuerdo al diagnóstico realizado por el Centro Mario Molina (CMM) en el 2015, el aire acondicionado representaba, en hoteles de 4 y 5 estrellas, hasta un $57 \%$ del consumo total de energía, iluminación $15 \%$, refrigeración $9 \%$ y habitaciones $3.67 \%$. Indicando que: "El uso de aire acondicionado llega a representar el 50\% del total de energía eléctrica en este tipo de establecimientos, en regiones cálidas el consumo de aire acondicionado llega a ser el $60 \%$ del consumo total de electricidad" (Centro Mario Molina [CMM], 2015, p. 54). Actualmente, aunado al reto de la utilización más eficiente de la energía por cuestiones de contaminación atmosférica en los destinos turísticos de México, se agrega otro elemento que conmina a los propietarios de los hoteles a mejorar la eficiencia, los costos crecientes que tiene el servicio en el país. Es decir, los propietarios de estos establecimientos están soportando costos cada vez más elevados en el servicio, incrementos que van del $20 \%$ al $60 \%$. Algunos presidentes regionales de la Asociación de Hoteles y Moteles en México, han llegado al extremo 
de tramitar amparos contra la Comisión Reguladora de Energía [CRE] (Ramírez, 2018; Reyna, 2018).

Siendo el turismo una actividad que demanda recursos energéticos y genera emisiones de $\mathrm{CO}_{2}$, es importante conocer los esfuerzos que este sector ha realizado en materia de eficiencia energética y contribuir de esta manera a las metas globales de los principales organismos internacionales. En este sentido se debe señalar que la tendencia mundial respecto a la eficiencia energética en hoteles se puede encontrar en el reporte Green Lodging Trends Report para el año 2017 (Greenview, 2017) ${ }^{11}$, el cual, es realizado anualmente y destacan prácticas de eficiencia energética relacionadas con el monitoreo del consumo de energía y el equipamiento eficiente en iluminación y controles digitalizados que permiten la reducción del consumo de energía.

\section{BENEFICIOS DE LA EFICIENCIA ENERGÉTICA EN EL SECTOR HOTELERO.}

En general, autores como Zabaloy, Recalde y Guzowski (2017) mencionan que "Una política que persiga como objetivo mejorar la EE (Eficiencia Energética) permite alcanzar la sostenibilidad energética y ambiental, en la medida en que se emplean tecnologías que consumen menos energía o igual cantidad, pero brindan mayores servicios energéticos. Por lo tanto, la EE puede ser vista como un recurso más, que asegura la sustentabilidad tanto del sistema económico como del ambiental".

Prasad y Singh (2015) mencionan que el turismo orientado a la reducción de $\mathrm{CO}_{2}$ es cada vez más importante en el desarrollo sustentable de la industria turística principalmente por el deseo de contribuir a la lucha contra el cambio climático y que es a través de la eficiencia energética que puede lograrse ese objetivo.

Es importante destacar que las prácticas encaminadas a la eficiencia energética en hoteles, tienen un impacto positivo en varios aspectos. Graci y Dodds (2008) distinguen siete beneficios relacionados con la operación de un hotel teniendo en consideración aspectos ambientales: 1) Ahorro en los costos de operación, 2) Mejor ventaja competitiva, 3) Mayor lealtad por parte de los empleados, 4) Mayor retención de clientes, 5) Cumplimiento normativo de leyes o reglamentos (locales, estatales, federales e internacionales), 6) Solventar el manejo de riegos y 7) La responsabilidad social.

De los beneficios mencionados, el de ahorro en costos de energía es el primer impulso por el cual los propietarios de los hoteles se ven incentivados a mejorar sus prácticas sustentables (Oluseyi et al., 2016; Prasad y Singh, 2015 y Bahadori-Jahromi, Rotimi, Mylona, Godfrey y Cook, 2017) específicamente aquellas relacionadas con la eficiencia energética, principalmente en cambios que permitan una disminución del uso de energía en sus diferentes operaciones.

11. Es un reporte generado mediante una encuesta por Greenview and Green Lodging News en colaboración con las principales cadenas mundiales de hoteles, destinos turísticos, líneas de cruceros e instituciones de investigación y certificación entre las que destacan Green Seal, Green Key Global, Foundation for Environmental Education, Travelife, Boston Green Tourism, entre otras. 
Sin embargo, aun cuando la eficiencia energética puede implicar un beneficio económico en el mediano y largo plazo, realizar los cambios necesarios en los equipos tiene un costo significativo en el corto plazo, pues se requiere una inversión para la adquisición de equipos más eficientes, convirtiéndose en una primera barrera para transmutar los hoteles en establecimientos sustentables. En este sentido el CMM (2015, p. 41) identifica tres umbrales de acceso al financiamiento para equipos de eficiencia energética:

Umbral 1: Usos energéticos simples que requieren poca capacitación y cuyos costos son bajos y accesibles. Iluminación y sustitución de luminarias.

Umbral 2: Adquisición y/o sustitución de equipos de mediano costo/inversión que requieren calificación técnica para su instalación y mantenimiento. Motores, aire acondicionado, equipos unitarios y refrigeración.

Umbral 3: Adquisición y/o sustitución de equipos mayores que requieren calificación técnica alta o de especialistas y, por tanto tienen altos costos y serían poco accesibles para una buena parte de los hoteles. Como son las calderas, recuperación de calor y sistemas de gestión de energía.

Se considera, a partir de la descripción anterior, que un alto porcentaje de los hoteles en este estudio tendrían incentivos para realizar cambios en la iluminación y la sustitución de luminarias, por su bajo costo y el beneficio que conlleva el ahorro en los costos de energía eléctrica. Sin embargo, lo señalado en los umbrales dos y tres, sería más difícil de encontrar en establecimientos medianos y pequeños, ya que los grandes hoteles, generalmente de 5 estrellas, son los que podrían realizar esta inversión. No obstante, estos hoteles tendrían que contemplar los incentivos, es decir, la posibilidad de aumentar sus precios, lo cual sería factible si existe la demanda suficiente por su servicio, o un ahorro de energía de tal dimensión que la inversión sea redituable en el mediano y largo plazo. Una posibilidad para lograr que se realicen las inversiones en la mayoría de los hoteles sería la regulación y los posibles incentivos fiscales por parte del gobierno federal.

En la literatura, se ha identificado el porcentaje de ahorro que representa en los costos de energía el implementar prácticas para realizar un uso eficiente de la misma. Por ejemplo, Graci y Dodds (2008), Rotimi et al. (2017) y Oluseyi et al. (2016) mencionan que puede reducirse el consumo de energía entre un $20 \%$ y un $40 \%$, sin afectar drásticamente el funcionamiento del hotel. En este sentido, Petrevska y Cingosky (2016) plantean estrategias que pueden reducir el uso de energía, como lo son, el cambio tecnológico, el cambio de comportamiento en relación con el uso de la energía en huéspedes y empleados, así como un cambio organizacional con la finalidad de establecer políticas de ahorro garantizando la comodidad para sus huéspedes. Dado que una falta de gestión en la operación de un hotel y una limitada conciencia de la conservación de energía por parte del personal que labora, resultado de una falta de formación, motivación e incentivos, impactan negativamente en las prácticas de eficiencia energética (Sheng et al., 2018; Arbelo, Pérez y Arbelo, 2016). 


\section{METODOLOGÍA}

Para el presente trabajo se procedió a ubicar los principales hoteles en el área de estudio, clasificándolos dentro de cinco zonas hoteleras: Madero-Playa Miramar, Madero, Tampico-Avenida Hidalgo, Tampico zona centro y Altamira. Según el INEGI (2017) para el año 2015 en Altamira existían 20 hoteles, en Ciudad Madero 49 y en Tampico 78, es decir 147 en total. Dado el ambiente de inseguridad que vive la entidad, es difícil que los hoteles proporcionen sus datos, aun tratándose de estudios científicos, por la desconfianza que ha creado esta situación. Sin embargo, se contó con la disposición de la Asociación de Hoteles del Sur de Tamaulipas A.C., organismo que proporcionó un listado de los hoteles miembros y el aval para poder realizar las encuestas. Por tanto, la población objetivo del presente trabajo está conformada por los miembros de la Asociación de Hoteles del Sur de Tamaulipas.

Para construir el cuestionario, el cual se recoge en el anexo 1, se tomaron como base los principales lineamientos para certificar los hospedajes verdes o sustentables relacionados con la eficiencia energética que proponen organismos como Green Lodging Program (Florida Department of Enviromental Protection [DEP], 2018) y la certificación Key Green Program (Foundation for Enviromental Education [FEE], 2016). Cabe señalar que el planteamiento teórico de estos organismos, aunque no lo señalen de manera explícita, es una visión intermedia entre la sustentabilidad débil y la fuerte (Williams y Millington, 2004), es decir, toman las propuestas que consideran más relevantes de ambas posiciones y las utilizan en sus estudios ${ }^{12}$. Dicho enfoque también se plantea en este trabajo al combinar el avance tecnológico, la concientización de dueños, gerentes y empleados, así como el cuidado del medio ambiente, entre otras situaciones que existen sobre la problemática y sus acciones futuras.

La encuesta fue estructurada en dos partes denominadas: caracterización socioeconómica y caracterización energética del establecimiento -ésta última, dividida en mantenimiento, equipamiento y construcción del establecimiento. Éstas se realizaron en dos momentos, por limitaciones económicas y por los tiempos de los gerentes o encargados de área de los hoteles. El primero se realizó en abril del 2018 y el segundo en agosto del mismo año.

Se diseñó una ruta de visitas de acuerdo a la ubicación de los establecimientos, para la optimización del combustible y tiempo en los traslados. A continuación se procedió a visitar cada uno de los hoteles para solicitar a sus recepcionistas entrevistar a la persona que podría contestar la encuesta. En la mayoría de los casos la disposición fue favorable. En dos casos la respuesta fue negativa, ya que las personas no se encontraban en esos momentos en el establecimiento. En cuatro casos hubo un rechazo directo a contestar la encuesta y en otros tres se solicitó dejar la encuesta para que el encargado la contestara, pero al final no la contestaron argumentando diferentes razones. Finalmente en dos casos, los hoteles no se encontraron en la dirección señalada en el listado. La población objetivo fue, de acuerdo a la información proporcionada por la asociación, de 36 hoteles, pero con lo antes señalado sólo se obtuvieron 25 encuestas. La información obtenida fue capturada en una base de datos y analizada utilizando el paquete estadístico Statistical Packing for Social Sciences (SPSS). El tamaño de muestra se

12. El avance tecnológico, pero también el límite en los recursos naturales. 
obtuvo estadísticamente tomando un nivel de confianza de $95 \%$ y un margen de error de $18 \%$, sin embargo el muestreo no fue aleatorio.

\section{RESULTADOS}

En una primera instancia, las encuestas se agrupan por el rango de tarifas que cobran los hoteles en pesos mexicanos, como se muestra en el cuadro 2.

Cuadro 2.- Características de los hoteles encuestados

\begin{tabular}{|c|c|c|c|c|}
\hline $\begin{array}{c}\text { Rango de tarifas promedio } \\
\text { por noche en pesos mexicanos } \\
(M X N)\end{array}$ & $\begin{array}{c}\text { Número } \\
\text { de hoteles }\end{array}$ & $\begin{array}{c}\text { Número de } \\
\text { habitaciones }\end{array}$ & Categoría & $\begin{array}{c}\text { Número de } \\
\text { trabajadores }\end{array}$ \\
\hline $300-600$ & 4 & 92 & 3 estrellas/sc* & 25 \\
\hline $601-1000$ & 6 & 376 & 3 y 4 estrellas & 148 \\
\hline $1001-1500$ & 10 & 855 & 4 estrellas/sc & 368 \\
\hline $1501-2000$ & 4 & 623 & 4 y 5 estrellas & 380 \\
\hline $2001-2500$ & 1 & 15 & 3 estrellas & 6 \\
\hline Total & $\mathbf{2 5}$ & $\mathbf{1 9 6 1}$ & *SC: Sin categoría & $\mathbf{9 2 7}$ \\
\hline
\end{tabular}

Fuente: Elaboración propia con datos de la encuesta aplicada a los hoteles.

Del total de hoteles encuestados, 10 se ubican en Ciudad Madero, 14 en Tampico y 1 en Altamira. Los hoteles que predominan son los que cuentan con la clasificación de 4 estrellas (56\%), distinción que otorga la Secretaría de Turismo ${ }^{13}$ y que junto con los de 3 estrellas conforman el $76 \%$ del total de la muestra. El mayor número de habitaciones lo ofrecen los hoteles con 4 y 5 estrellas, situación estrechamente relacionada al número de empleados que laboran en ellos como se observa en el cuadro 3.

Para observar si existe algún seguimiento en el gasto de energía que realizan estos establecimientos se preguntó si llevaban a cabo un registro de manera periódica de su consumo de energía obteniendo los siguientes resultados:

13. El sistema de clasificación hotelera es un mecanismo de autoevaluación que agrupa los principales servicios que determinan, mediante una tabla de puntajes, la categoría del establecimiento de hospedaje que va de 1 a 1300 puntos y va de 1 a 5 estrellas incluyendo la denominación sin categoría (Diario Oficial de la Federación, 2016). 
Gráfica 4.- Registro del consumo energético en los hoteles

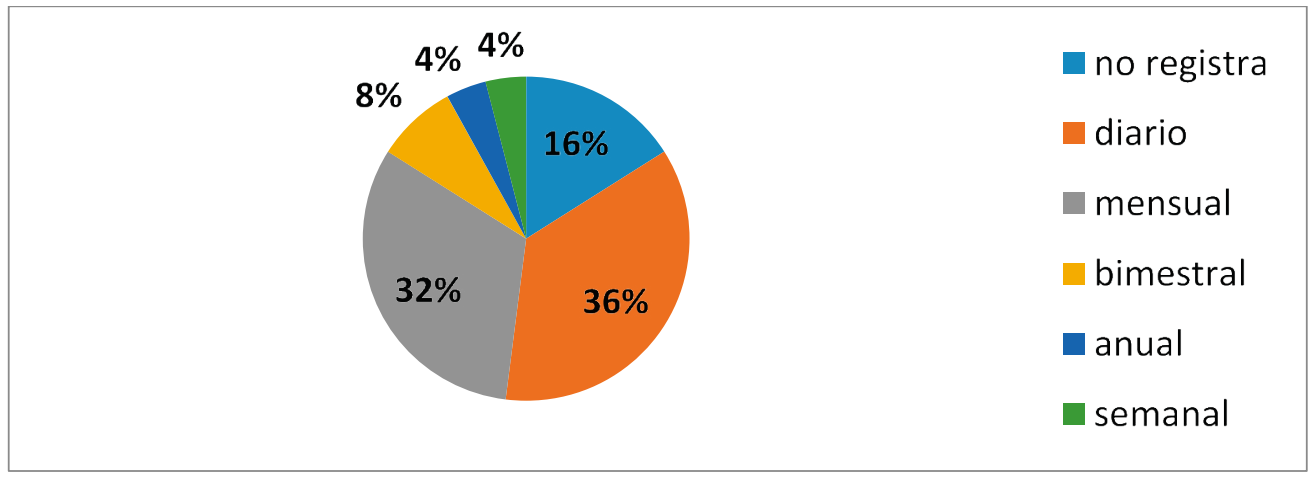

Fuente: Elaboración propia con datos de la encuesta aplicada a los hoteles.

El 84\% señaló que sí lo realizaba, de éstos, el 68\% lo hacía de manera diaria o mensual. El $16 \%$ indicó no llevar a cabo esta práctica. En este sentido, cabe destacar que el $56 \%$ de los establecimientos señaló no contar con medidores en las áreas de alto consumo de energía, como serían la cocina, el gimnasio o climatización central y solamente el $8 \%$ del total están equipados con estos medidores en la mayoría de las áreas antes mencionadas. Por otra parte, se preguntó si reciben visitas por parte de autoridades locales, estatales o federales en relación con la eficiencia energética del hotel, encontrando que el $48 \%$ mencionaron haber recibido alguna visita con este fin, destacando las visitas federales como las más recurrentes. En relación a lo anterior, el $64 \%$ de los establecimientos señalaron tener una política formal respecto a los mecanismos utilizados para el ahorro de energía.

Lo siguiente fue conocer el uso de energía renovable o eco certificada en los hoteles. Al respecto, ninguno de los hoteles encuestados manifestó que la energía renovable sea su principal fuente de energía para la operación del mismo y solamente un hotel mencionó su uso en combinación con la electricidad y el gas de red. Del total de hoteles, sólo el $12 \%$ de los establecimientos señalaron usar fuentes de energía renovable, esto de manera parcial, dado que, indicaron que se utilizaban en menos del $50 \%$ del consumo total de energía, utilizando principalmente electricidad y gas, ya sea gas natural de red o lp para su funcionamiento. En relación a las fuentes de energía utilizadas para la iluminación y la refrigeración se encontró que en el $98 \%$ (en promedio) de los casos se empleaba la electricidad. Para la cocción de los alimentos y producción de agua caliente utilizaron el gas en sus dos presentaciones en el 91.1\% (en promedio) de los casos -solamente un hotel emplea fuentes de energía renovables para la producción de agua caliente-.

En relación al mantenimiento del hotel, se encontró que, de manera general, se realiza mantenimiento preventivo, lo que debería repercutir en un ahorro de energía importante para el hotel. Se destaca en este sentido que en el $84 \%$ de los hoteles la tubería de agua caliente se encuentra aislada para evitar el uso excesivo de fuentes de calor. En cuanto a la revisión y limpieza de extractores de ventilación, así como a la sustitución y/o limpieza de los filtros de las campanas de cocina, prácticamente el $100 \%$ de los establecimientos afirman realizar algún tipo de inspección y limpieza, destacando la revisión del sistema de ventilación, el cual realizan el $68 \%$ de los hoteles, al menos una vez cada tres meses. En cuanto a las reparaciones en 
los sistemas de ventilación y/o aire acondicionado, el 66.7\% de los establecimientos realizó de una a seis reparaciones anuales, lo cual, también podría ser indicativo de la obsolescencia de muchos de los equipos utilizados, lo que pudiera estar generando mayores costos de operación. En lo referente a la impermeabilización en techos, se destaca que el $84 \%$ de los establecimientos señalan hacerlo de manera periódica, en particular el $57 \%$ de ese porcentaje lo realizan cada cinco años, un tiempo estándar en la vida útil de los impermeabilizantes.

En cuanto a las medidas relacionadas al mejoramiento en el equipamiento para una mejor eficiencia energética, se encontró que el $80 \%$ cuenta con tecnología LED en la iluminación, aunque en la mayoría de los casos de forma parcial, pues únicamente $36 \%$ de ellos están equipados con esta tecnología al 100\%. También se preguntó si contaban con equipos ahorradores, automatizados, aislantes o certificados, encontrando tanto respuestas positivas como negativas. Al preguntar si se contaba con sensores de movimiento o apagado controlado en la iluminación exterior, el 65.2\% mencionó que sí, sin embargo cuando se realizó la misma pregunta para las áreas comunes interiores (salones, corredores, bares, gimnasios o restaurantes) y habitaciones, los resultados arrojaron que el $75 \%$ no contaba con este equipamiento en las áreas comunes y el $68 \%$ tampoco lo tenía en las habitaciones. Por su parte, también se encontró que solo el $24 \%$ de los hoteles están equipados con llave inteligente o detector de movimiento, siendo principalmente los hoteles de 4 y 5 estrellas los que cuentan con este equipo.

Dentro del equipamiento, una de las acciones que ayuda a reducir la transferencia del calor en las habitaciones es el aislante térmico en las ventanas y/o la política de mantener restringida la apertura de éstas. Para el caso de los hoteles encuestados, el 52\% indicaron tener la totalidad de sus ventanas aisladas, lo cual, se podría considerar un bajo porcentaje dado el tipo de clima que impera en la región. Además, hay 13 hoteles que no contemplan la restricción en cuanto a la apertura de ventanas y de éstos, 10 no cuentan con algún sistema que detecte la apertura de las ventanas para apagar de manera automática la climatización de la habitación. Por último, en lo referente al uso de sistemas automáticos ahorradores de energía en áreas como las piscinas, los gimnasios y los restaurantes, entre otros, los resultados obtenidos estipulan que el $56 \%$ de los establecimientos de hospedaje no cuentan con ellos y únicamente el $8 \%$ está equipado casi en su totalidad. En el caso específico de las tinas de hidromasaje, saunas y piscinas solo el $20 \%$ cuentan con algún temporizador para el ahorro de energía.

En lo que se refiere a los principios que fueron considerados en la construcción o remodelación de los hoteles relacionados a la eficiencia energética, el 40\% manifestó haber tomado en cuenta el uso efectivo de la sombra en la construcción del inmueble, para la reducción de los costos por climatización. Por su parte el $12 \%$ de estos inmuebles manifestó contar con muros verdes y ninguno de ellos manifestó contar con techos verdes, una práctica que ayuda a reducir el consumo de energía en estos inmuebles, al ser una técnica que reduce la temperatura al interior de los mismos.

Para obtener un panorama general de los resultados encontrados, se realizó un índice de eficiencia energética, para sintetizar información en relación al cumplimiento de las distintas medidas sustentables e identificar su relación con las características del establecimiento. En el cuadro 3 se presentan los resultados del cumplimiento de 35 lineamientos contemplados en la encuesta. Para obtenerlo, se procedió a otorgar un puntaje a cada uno de ellos, siendo 2 
puntos para quienes cumplían en su totalidad con la condición, 1 punto para quienes cumplían de manera parcial ${ }^{14}$ y sin asignación de puntaje para quienes no cumplían con el lineamiento. Al ser 70 el puntaje máximo posible, se procedió a obtener el porcentaje de cumplimiento y el orden jerárquico correspondiente.

Cuadro 3.- Índice de eficiencia de hoteles encuestados

\begin{tabular}{|c|c|c|}
\hline Hotel & Puntos de eficiencia & $\begin{array}{l}\text { Porcentaje de } \\
\text { cumplimiento }\end{array}$ \\
\hline $\mathbf{I}$ & 53 & 75.71 \\
\hline II & 50 & 71.43 \\
\hline III & 49 & 70.00 \\
\hline IV & 46 & 65.71 \\
\hline $\mathbf{v}$ & 43 & 61.43 \\
\hline VI & 41 & 58.57 \\
\hline VII & 40 & 57.14 \\
\hline VIII & 40 & 57.14 \\
\hline IX & 39 & 55.71 \\
\hline$x$ & 38 & 54.29 \\
\hline $\mathbf{X I}$ & 35 & 50.00 \\
\hline XII & 35 & 50.00 \\
\hline XIII & 34 & 48.57 \\
\hline XIV & 34 & 48.57 \\
\hline XV & 33 & 47.14 \\
\hline $\mathbf{X V I}$ & 33 & 47.14 \\
\hline XVII & 28 & 40.00 \\
\hline XVIII & 27 & 38.57 \\
\hline $\mathbf{X I X}$ & 26 & 37.14 \\
\hline$x x$ & 25 & 35.71 \\
\hline $\mathbf{X X I}$ & 25 & 35.71 \\
\hline XXII & 24 & 34.29 \\
\hline XXIII & 23 & 32.86 \\
\hline XXIV & 22 & 31.43 \\
\hline$X X V$ & 20 & 28.57 \\
\hline
\end{tabular}

Fuente: Elaboración propia con datos de la encuesta aplicada a los hoteles.

En el cuadro anterior se puede observar de manera general, un bajo porcentaje de cumplimiento de los lineamientos de eficiencia energética considerados por Green Lodging Program y el Key Green Program en los establecimientos encuestados. Únicamente el $20 \%$

14. Un ejemplo para determinar el cumplimiento parcial puede referirse a la pregunta ¿Cuentan las ventanas de las habitaciones con algún tipo de aislante térmico? Encontrando respuestas como todas, algunas y ningunas, por lo cual se le asigna un punto a la respuesta "algunas". 
de los hoteles obtuvieron porcentajes aceptables, teniendo en cuenta un umbral de más de $60 \%$ de cumplimiento. De éstos, tres son de 4 estrellas y dos de 5 estrellas, lo cual, permite inferir que existe una relación directa entre la categoría del hotel y la implementación de medidas de eficiencia energética, es decir, que los hoteles con mayor número de habitaciones e ingresos tendrían mayor facilidad para realizar inversiones en equipos más eficientes $\mathrm{y} / \mathrm{o}$ contemplar políticas formales sobre las prácticas eficientes en sus instalaciones. Al observar el porcentaje de cumplimiento de los 10 primeros hoteles se tiene que: Siete de ellos son de 4 estrellas, dos de 5 estrellas y 1 sin categoría ${ }^{15}$ (esta última una observación que se podría considerar atípica). En relación a la ubicación geográfica de estos 10 hoteles, se destaca que ocho se encuentran en el municipio de Tampico, uno en Ciudad Madero y otro más en Altamira lo cual representa, en términos de oferta respecto al número de habitaciones, el $58 \%$ del total. Otros resultados generales que se desprenden de los datos obtenidos, son las prácticas que más y menos llevan a cabo los establecimientos de la zona de estudio, destacando, de manera jerárquica, las 10 principales, enlistadas en el cuadro 4.

\section{Cuadro 4.- Prácticas de eficiencia energética en hoteles encuestados}

\begin{tabular}{|c|c|c|c|}
\hline No. & $\begin{array}{l}\text { Prácticas de eficiencia energética más } \\
\text { recurrentes. }\end{array}$ & No. & $\begin{array}{l}\text { Prácticas de eficiencia energética menos } \\
\text { recurrentes. }\end{array}$ \\
\hline 1 & Política de lavado de sábanas en agua fría. & 1 & $\begin{array}{l}\text { Equipamiento de sistema de recuperación de } \\
\text { calor reutilizable en otras áreas. }\end{array}$ \\
\hline 2 & Tubería de agua caliente aislada. & 2 & $\begin{array}{l}\text { Piscinas y tinas cubiertas cuando no están en } \\
\text { uso. }\end{array}$ \\
\hline 3 & $\begin{array}{l}\text { Equipo de cómputo equipado con sistema de } \\
\text { ahorro de energía. }\end{array}$ & 3 & Uso de fuentes de energía renovables. \\
\hline 4 & $\begin{array}{l}\text { Cerrado parcial de instalaciones en temporada } \\
\text { baja. }\end{array}$ & 4 & $\begin{array}{l}\text { Equipamiento de sistema de apagado } \\
\text { automático en habitaciones. }\end{array}$ \\
\hline 5 & Ajuste de temperatura estándar $22^{\circ}$. & 5 & $\begin{array}{l}\text { Instalación de muros y techos verdes en las } \\
\text { instalaciones. }\end{array}$ \\
\hline 6 & Impermeabilización de instalaciones. & 6 & $\begin{array}{l}\text { Equipamiento de temporizadores en piscinas, } \\
\text { tinas y saunas. }\end{array}$ \\
\hline 7 & Limpieza y sustitución de filtros de campana. & 7 & $\begin{array}{l}\text { Equipamiento de encendido de campanas de } \\
\text { cocina automático. }\end{array}$ \\
\hline 8 & Política formal de ahorro de energía. & 8 & $\begin{array}{l}\text { Equipamiento de sistema de apagado } \\
\text { automático en habitaciones desocupadas. }\end{array}$ \\
\hline 9 & Revisión del sistema de ventilación. & 9 & $\begin{array}{l}\text { Equipamiento de apagado automático en } \\
\text { salones y corredores. }\end{array}$ \\
\hline 10 & $\begin{array}{l}\text { Sistema de ahorro de energía en iluminación } \\
\text { exterior. }\end{array}$ & 10 & $\begin{array}{l}\text { Equipamiento de ventiladores de optimización } \\
\text { energética. }\end{array}$ \\
\hline
\end{tabular}

Fuente: Elaboración propia con datos de la encuesta aplicada a los hoteles.

15. De acuerdo al reglamento de la Ley General de Turismo en México (2015), es obligación de los establecimientos dedicados al alojamiento temporal, realizar la autoevaluación para obtener la Constancia de Clasificación Hotelera. Dicha constancia contiene 6 rubros, que van de 1 a 5 estrellas y el rubro denominado "Sin categoría" la cual queda a consideración del prestador de servicio clasificarse bajo esta opción. Por lo tanto, no significa expresamente que quienes optan por clasificarse "sin categoría" sean inferiores a los que se clasifican en las opciones de 1 a 5 estrellas. 
Del análisis del cuadro anterior se puede determinar que las prácticas más recurrentes que se llevan a cabo, son las que conllevan poca inversión de los hoteles, por el contrario, las prácticas que menos se llevan a cabo, requieren de una inversión en equipamiento del hotel, generando mayores costos de inversión para los propietarios.

\section{CONCLUSIONES}

El presente estudio muestra los resultados sobre el fomento de políticas y prácticas que se hacen de eficiencia energética en los hoteles de los municipios de Altamira, Ciudad Madero y Tampico en el estado de Tamaulipas en México, basados en los lineamientos que para certificar los hospedajes en este aspecto proponen organismos como Green Lodging Program (Florida Department of Enviromental Protection [DEP], 2018) y la certificación Key Green Program (Foundation for Enviromental Education [FEE], 2016), obteniéndose también a partir de ellos un índice sobre la eficiencia energética en los hoteles de la muestra. Además de que dadas las condiciones del destino, pueda servir como ejemplo o no a otros destinos de similares características para el desarrollo de esta clase de actividades, en principio buscando la conservación de energía pero teniendo como aspiración la búsqueda de lo que debiera ser un desarrollo sustentable.

La investigación encontró que existen disparidades muy marcadas entre los establecimientos estudiados, principalmente económicas, pero también por el tipo de demanda que enfrentan y su ubicación. Es por ello que existen establecimientos incorporados a cadenas hoteleras internacionales que cumplen con la mayoría de las medidas de eficiencia energética estudiadas. En contraposición, los hoteles nativos o más longevos son los que incumplen muchas de ellas y requieren políticas o acciones que los ayuden a transitar hacia el uso de equipos más eficientes que les permitan ser más competitivos y sustentables en su operación. Desde un punto de vista meramente económico ${ }^{16}$ y de alguna forma desde la sustentabilidad débil, los resultados antes descritos tendrían una prescripción directa, la competencia por el mercado debiera dejar fuera a todos los hoteles que no puedan incorporar la tecnología para ser más eficientes y dejar su parte del mercado para aquellos que si lo pueden hacer (los hoteles de cadenas internacionales). Pero no es una solución compatible con el desarrollo sustentable, pues en este también se debe considerar la preservación de la cultura nativa y la equidad entre los pobladores de la región. En este sentido son los hoteles más pequeños los que preservan algunas tradiciones locales, como puede ser tener una cocina más típica y utilizar insumos locales, entre otras. Si se considera la equidad, no se les puede dejar en agonía hasta cerrar, porque son generadores de ingresos y de puestos de trabajo, además que demandan insumos de la región para su funcionamiento. Por tanto, se les debe apoyar para que, desde sus posibilidades, incorporen la eficiencia energética, situación que en vez de darse desde el cambio tecnológico inmediato, se podría implementar desde la concientización en el cuidado y ahorro de las energías desde el propietario hasta los empleados y huéspedes, para que en el mediano y largo plazo incorporen algunas mejoras técnicas con el apoyo del gobierno local.

16. Ligado a la principal corriente que proclama la preponderancia de los mercados y la competencia. 
No obstante, se puede afirmar que todos los hoteles encuestados han implementado prácticas de eficiencia energética, sin embargo, de acuerdo al índice desarrollado, los esfuerzos para realizar esta práctica son muy variables. Pareciera ser que los umbrales 2 y 3 , como se predecía, no son fáciles de alcanzar para los hoteles regionales. En cambio, los hoteles internacionales tienen mayores posibilidades de alcanzarlos.

Es importante remarcar que los hoteles ubicados en el municipio de Ciudad Madero -donde se encuentra el destino turístico de sol y playa- son los que tienen índices de eficiencia energética más bajos. De los 10 hoteles encuestados en este municipio solo uno pertenece a los 10 primeros en el indicador de eficiencia. El municipio de Tampico presenta un mejor desempeño, ya que de 14 hoteles encuestados, 8 se encuentran en los primeros diez lugares. Por último, en relación a Altamira puede decirse que el único hotel encuestado se encuentra entre las diez primeras posiciones. En el cuadro 5 se puede observar el porcentaje de cumplimiento promedio por municipio.

Cuadro 5.- Índice de eficiencia por municipios (promedio)

\begin{tabular}{|c|c|}
\hline Ciudad & Porcentaje de cumplimiento (Promedio) \\
\hline Tampico & $53.98 \%$ \\
\hline Ciudad Madero & $41.14 \%$ \\
\hline Altamira & $65.71 \%$ \\
\hline
\end{tabular}

Fuente: Elaboración propia con datos de la encuesta aplicada a los hoteles.

Como se mencionó al inicio del trabajo, uno de los mayores desafíos para el sector hotelero en la región es controlar la climatización artificial. Los resultados del estudio muestran que dentro de las medidas de eficiencia energética implementadas por los hoteles de la muestra, una de las más relevantes es la estandarización de una temperatura promedio de 220 , práctica que es llevada a cabo por el $68 \%$ de los hoteles. Además en relación al uso de los aires acondicionados, el $76 \%$ de los hoteles señalan cerrar las áreas no utilizadas en temporada baja. Según estimaciones de fuentes consultadas del sector hotelero en la zona de estudio, entre el $15-20 \%$ de los costos totales de la operación del hotel, provienen del sector energético, los cuales son muy superiores a la literatura, que plantea costos "entre un 3 y $6 \%$ " (Bohdanowicz et al., 2001, p. 2). En este sentido, se estarían triplicando los costos de energía de un hotel en la zona de estudio, abriendo la posibilidad de plantear políticas de eficiencia energética para contrarrestar el consumo intensivo de energía en esta actividad económica.

Al respecto, se realizó una búsqueda en los documentos oficiales de los tres municipios de la zona de estudio y en el estado de Tamaulipas, encontrando en los Programas de Ordenamiento Territorial (POT) de Ciudad Madero y en el POT metropolitano la misma información. En el capítulo seis, denominado acciones estratégicas específicas de desarrollo urbano organizadas en proyectos particulares, en la estrategia de infraestructura, se señala: "La sustitución de equipos para el ahorro de energía en iluminación y aire acondicionado eficiente en edificios públicos y grandes edificaciones, tal como centros comerciales, complejos de oficinas y hoteles" (Programa de Ordenamiento Territorial [POT], 2016 p.166). En caso de no llevarse a cabo la medida, se incrementaría la huella ecológica para las futuras generaciones de la 
zona, debido a la utilización de equipos obsoletos o de consumo de energía alto. Sin embargo, aunque esto parece una política acertada, cabe destacar que las autoridades, tanto estatales como municipales, no contemplan implementarla en el corto plazo, ya que está catalogado como un proyecto de prioridad baja. Esto pone en evidencia que no se están atendiendo los daños que está causando y que causará no considerar su importancia para cuestiones sociales como la salud, económicas como la competencia y ecológicas como el daño que se causa a la naturaleza existente en la región.

Por último, se puede observar que las políticas y prácticas sobre eficiencia energética que señalan tener los hoteles de la muestra son muy dispares y existen áreas de oportunidad para mejorarlas desde los gobiernos locales, el federal y los dueños de los establecimientos, para propiciar un desarrollo sustentable en estos destinos. A su vez, se debe señalar que futuros estudios sobre esta temática para la zona de estudio debieran incluir información primaria sobre costo y cantidad de energía con la finalidad de correlacionarlo con factores que inciden de manera directa con el consumo energético. Estas líneas de investigación se han abordado en otras latitudes y puede llegar a ser de gran utilidad para las partes interesadas directamente como lo son propietarios y gobierno. Al mismo tiempo, en un trabajo futuro se podría replicar este análisis con la totalidad de los hoteles para obtener un panorama más general de la problemática. En el presente trabajo esto no fue posible debido a las condiciones de seguridad en el estado y a la falta de financiamiento.

\section{REFERENCIAS BIBLIOGRÁFICAS}

Agencia Internacional de Energía (2015). Indicadores de Eficiencia Energética: Bases esenciales para establecimiento de políticas. Francia. Pp. 182. Recuperado de https://www.iea. org/publications/freepublications/publication/EnergyEfficiencyVespagnol_epdf.pdf.

Arbelo, A., Pérez-Gómez, P., y Arbelo-Pérez, M. (2016). Cost efficiency and its determinants in the hotel industry. Tourism Economics, 23(5), 1056-1068. DOI:10.1177/1354816616656419

Bahadori-Jahromi, A., Rotimi, A., Mylona, A. Godfrey, P. y Cook, D. (2017). Impact of Window Films on the Overall Energy Consumption of Existing UK Hotel Buildings. Sustainability, 9 (5), 731. DOI: 10.3390/su9050731.

Bianco, V., Righi, D., Scarpa, F. y Tagliafico, L. (2017). Modeling energy consumption and efficiency measures in the Italian hotel sector. Energy and Buildings 149 (2017), 329-338. DOI: 10.1016/j.enbuild.2017.05.077.

Bohdanowicz, P., Churie-Kallhauge, A., y Martinac, I. (2001). Energy-Efficiency and Conservation in Hotels-Towards Sustainable Tourism. Trabajo presentado en 4th International Symposium on Asia Pacific Architecture, University of Hawai'l at Manoa, Honolulu, Hawaii.

Cámara de Diputados del H. Congreso de la Unión. (6 de julio del 2015). Artículo Transitorio décimo séptimo. Reglamento de la Ley de Federal de Turismo. DOF: <06/07/2015>. Recuperado de http://www.sectur.gob.mx/pdf/0216/Reglamento Ley General Turismo.pdf.

Centro Mario Molina (2015). Hacia una Estrategia Nacional de Eficiencia Energética para las Edificaciones de Hoteles y Restaurantes. México. Recuperado de http://www.sectur. gob.mx/2017/TuristismoSustentable/CambioClimatico/ll.2.4\%20Hacia\%20una\%20 Estrategia\%20Nacional\%20de\%20Eficiencia\%20Energe\%CC\%81tica\%202015.pdf. 
Comisión Federal de Electricidad CFE. (2007). Consumo de energía eléctrica por entidad federativa. Anexo estadístico de informe de gobierno. Recuperado de http://calderon. presidencia.gob.mx/informe/primer/pdf_excel/AnexoEstadistico.pdf.

Crece 8.51\% el turismo de verano en Tamaulipas. (22 de agosto del 2018). Excelsior. Recuperado de https://www.excelsior.com.mx/nacional/ crece-851-el-turismo-de-verano-en-tamaulipas/1260219.

Cruz, V. (5 de octubre del 2017). Enseñan ahorrar energía a empresarios tamaulipecos. Milenio. Recuperado de https://www.milenio.com/negocios/ensenan-ahorrar-energia-aempresarios-tamaulipecos.

Dachary, A. y Arnaiz, S. (2006). EL ESTUDIO DEL TURISMO ¿Un paradigma en formación? Estudios y Perspectivas en Turismo, 15 (2), 179-193. ISSN 0327-5841. Recuperado de http://www.redalyc.org/articulo.oa?id=180713891004 .

Diario Oficial de la Federación (2016). Acuerdo por el que se emiten los Lineamientos del Sistema de Clasificación Hotelera. México: Diario Oficial de la Federación. Recuperado de http://sch.sectur.gob.mx:8080/SCHWEB/resources/LINEAMIENTOS-SCH- DOF.pdf.

Florida Department of Environmental Protection (2018). Green Lodging Program Designation Application. USA. Recuperado de https://floridadep.gov/sites/default/files/FGLP\%20 \%20DESIGNATION APPLICATION 201800 0.pdf.

Foundation for Environmental Education (2016). Green Key hotel criteria and explanatory notes 1 July 2016-31 December 2020. Dinamarca. Recuperado de https:// static1.squarespace.com/static/55371f97e4b0fce8c1ee4c69/t/5ae84e818a922d40d2bcc16b/1525173893129/Green+Key+hotel+criteria+explanatory+no tes+2016-2020+revision+2018.pdf.

Gobierno de la República (2013). Plan Nacional de Desarrollo 2013-2018. México. Recuperado de http://pnd.gob.mx/.

Gobierno Municipal de Ciudad Madero (2013). Plan Municipal de Desarrollo Cd. Madero 2013-2016. México. Recuperado de: https://docplayer.es/37052058-Plan-municipal-de-desarrollo-cd-madero-plan-municipal-de-desarrollo-cd-madero.html.

Graci, S. y Dodds, R. (2008). Why Go Green? The Business Case for Environmental Commitment in the Canadian Hotel Industry. Anatolia: An International Journal of Tourism and Hospitality Research, 19:2, 251-270. Doi: 10.1080/13032917.2008.9687072.

Greenview (2017). Green Lodging Trends Report 2017. Recuperado de http://www. greenlodgingnews.com/wp-content/uploads/2017/09/Green-Lodging-TrendsReport-2017 Final.pdf.

Idahosa, L., Marwa, N. y Akotey, J. (2017). Energy (Electricity) Consumption in South African Hotels: A Panel Data Analysis. Energy and Buildings, (156), 207-217 DOI: 10.1016/j. enbuild.2017.09.051

Instituto Nacional de Estadística y Geografía INEGI. (2015). “Estadísticas a propósito del... día mundial del turismo (27 de Septiembre)". Instituto Nacional de Estadística y Geografía. Aguascalientes, Ags. México. 23 de septiembre del 2015. Pp.11. Recuperado de http:// www.inegi.org.mx/saladeprensa/aproposito/2015/turismo0.pdf.

Instituto Nacional de Estadística y Geografía INEGI (2017). Anuario estadístico y geográfico de Tamaulipas 2017. México. Pp.551. Recuperado de http://www.datatur.sectur.gob.mx/ itxef docs/tams anuario pdf.pdf. 
Lenzen, M., Sun, Y., Faturay, F., Ting, Y., Geschke, A., y Malik, A. (2018). The carbon footprint of global tourism. Natura Climate, 8 (6), 522-528. Doi: 10.1038/s41558-018-0141-x

Macías, T. (25 de julio del 2017). Tamaulipas arroja un 53\% de ocupación turística. El Financiero. Recuperado de https://www.elfinanciero.com.mx/monterrey/ tamaulipas-arroja-un-53-de-ocupacion-turistica.

Molina, A., Velarde, H., Borroto, A., Santiesteban, C., y Monteagudo, J. (2017). Nuevos índices de consumo energético para hoteles tropicales. Ingeniería Energética, XXXVIII (3), 198207. Recuperado de http://www.redalyc.org/articulo.oa?id=329152934005.

OECD (2017), Tourism Policy Review of Mexico, OECD Studies on Tourism, OECD Publishing, Paris.http://dx.doi.org/10.1787/9789264266575-en. Recuperado de https://www. datatur.sectur.gob.mx/SiteAssets/SitePages/DocsPubs/TOURISM POLICY REVIEW MEXICO.pdf.

Olcina, J. (2012). Turismo y cambio climático: una actividad vulnerable que debe adaptarse. Investigaciones Turísticas, (4), 1-34. Recuperado de https://rua.ua.es/dspace/bitstream/10045/25736/1/Investigaciones Turisticas 4 01.pdf.

Oluseyi, P., Babatunde, O. y Babatunde, O. (2016). Assessment of energy consumption and carbon footprint from the hotel sector within Lagos, Nigeria. Energy and Buildings, (118), 106-113. DOI: 10.1016/j.enbuild.2016.02.046

Organización Mundial del Turismo, Programa de Naciones Unidad para el Medio Ambiente y Organización Mundial Meteorológica (2008). Climate Change and Tourism- Responding to Global Challenges. España. Recuperado de https://sdt.unwto.org/sites/all/files/ docpdf/climate2008.pdf.

Organización Mundial del Turismo (2017). Ranking Mundial del Turismo Internacional. En Barómetro OMT -Volumen 15, agosto de 2017-Anexo Estadístico. Recuperado de http://www.datatur.sectur.gob.mx/SitePages/RankingOMT.aspx.

Organización Mundial del Turismo (2018a). El turismo internacional supera las expectativas en los primeros meses de 2018. Comunicado de prensa. Madrid, España. 25 de junio del 2018. Recuperado de http://media.unwto.org/es/press-release/2018-06-25/ el-turismo-internacional-supera-las-expectativas-en-los-primeros-meses-de-2.

Organización Mundial de Turismo (2018b). 2017: nuevo año récord según a la visión panorámica sobre el turismo. Comunicado de prensa. Madrid, España. 27 de agosto del 2018. Recuperado de http://www2.unwto.org/es/press-release/2018-08-27/2017-nuevoano-record-segun-la-vision-panoramica-sobre-el-turismo.

Organización Mundial de Turismo (2018c). La OMT presenta una plataforma en línea para impulsar la consecución de los ODS a través del turismo. Comunicado de prensa. Madrid, España. 23 dejulio del 2018. Recuperado de http://media.unwto.org/es/press-release/2018-07-23/ la-omt-presenta-una-plataforma-en-linea-para-impulsar-la-consecucion-de-los

Organización Mundial delTurismo (2019a). Resultados del turismointernacional2018yperspectivas2019. Recuperado dehttp://cf.cdn.unwto.org/sites/all/files/pdf/unwto barometer jan19 presentation sp.pdf

Organización Mundial del Turismo (2019b). Cifras claves del turismo. Infografía. Recuperado de http://cf.cdn.unwto.org/sites/all/files/pdf/unwto key figures barom may2019 sp.pdf. 
Paredes, M. (28 de agosto de 2018). México, el sexto país en el mundo con más visitas en el 2017. Excélsior. Recuperado de https://www.excelsior.com.mx/nacional/ mexico-el-sexto-pais-en-el-mundo-con-mas-visitas-en-2017/1261242.

Petrevska, B. y Cingosky, V. (2016). Energy efficiency practices: assessment of Ohrid hotel industry. Horizons 20(A), 511-520 December 2016. DOI: 10.20544/HORIZONS.A.20.1.17.P34.

Pieri, S., Tzouvadakis, I. y Santamouris, M. (2015). Identifying energy consumption patterns in the Attica hotel sector using cluster analysis techniques with the aim of reducing hotels' $\mathrm{CO}_{2}$ footprint. Energy and Buildings 94 (2015), 252-262. DOI: 10.1016/j. enbuild.2015.02.017.

Prasad, K. y Singh, A. (2015). Towards Low Carbon Hotels in the Pacific Region: A Study of Energy Consumption and Efficiency in Hotels Using Models Based on Energy Performance Indicators. Climate Change in the Asia-Pacific Region, 357-374. DOI: 10.1007/978-3-319-14938-7_21

Programa de Naciones Unidad para el Medio Ambiente (PNUMA), Gobierno del Estado de Tamaulipas y Secretaría de Medio Ambiente y Recursos Naturales (SEMARNAT). (2015). Perspectivas del Medio Ambiente y Cambio Climático en el Medio Urbano: ECCO Zona Conurbada del Sur de Tamaulipas. México. Recuperado de http://www.une.edu.mx/ ECCO.pdf.

Ramírez, R. (12 de septiembre de 2018). Van hoteleros por amparo contra tarifas de "luz". Vanguardia. Recuperado de https://vanguardia.com.mx/articulo/ van-hoteleros-por-amparo-contra-tarifas-de-luz.

Republicano Ayuntamiento de Ciudad Madero (Jueves 29 de septiembre de 2016). Actualización del Programa Municipal de Ordenamiento Territorial y Desarrollo Urbano de Cd. Madero, Tamaulipas 2016. Ciudad Victoria Tamaulipas. Periódico Oficial del Estado de Tamaulipas. Tomo CXLI número 117. Recuperado de http://po.tamaulipas.gob.mx/ wp-content/uploads/2016/09/cxli-117-290916F-ANEXO.pdf

Reyna, J. (20 de septiembre del 2018). Se unen hoteleros contra cobros excesivos de la CFE. La Jornada. Recuperado de https://www.jornada.com.mx/ultimas/2018/09/20/ se-unen-hoteleros-contra-cobros-excesivos-de-la-cfe-7379.html.

Rotimi, A., Bahadori-Jahromi, A., Mylona, A., Godfrey, P. y Cook, D. (2017). Estimation and Validation of Energy Consumption in UK Existing Hotel Building Using Dynamic Simulation Software. Sustainability 9(8), 1391. DOI: 10.3390/su9081391

Secretaría de Energía (2016). Balance Nacional de Energía 2016. Subsecretaría de Planeación y Transición Energética, Dirección General de Planeación e Información Energéticas, México. Recuperado de https://www.gob.mx/cms/uploads/attachment/file/288692/ Balance Nacional de Energ a 20162 .pdf

Secretaría del Medio Ambiente y Recursos Naturales SEMARNAT (2013). Programa de Playas Limpias. Ciudad Madero, Tamaulipas, México. Recuperado de http://www.semarnat. gob.mx/playas/playas limpias/destinos/ciudad-madero.

Secretaría del Medio Ambiente y Recursos Naturales SEMARNAT (2017). Entrega las primeras nueve banderas blancas con ese emblema. Comunicado.12 de junio del 2017. Recuperado de https://www.gob.mx/semarnat/prensa/ lanza-semarnat-distintivo-de-playas-limpias-sustentables?idiom=es. 
Secretaría del Medio Ambiente y Recursos Naturales SEMARNAT (2019). Resultados de calidad de agua de mar: Ciudad Madero, Tamaulipas. Recuperado de https://apps1.semarnat. gob.mx:8443/dgeia/gob-mx/playas/destinos/ciudad-madero.html.

Secretaría de Turismo SECTUR (2013). Programa Sectorial de Turismo (PROSECTUR 2013-2018). DOF: 13/12/2013. Recuperado de http://www.sectur.gob.mx/PDF/PlaneacionTuristica/ Prosectur 2013 2018.pdf.

Secretaría de Turismo SECTUR (2018). Información Turística por Entidad Federativa. Portal del Sistema Nacional de Información Estadística y Geográfica de Turismo SNIEGT. Recuperado de: https://www.datatur.sectur.gob.mx/ITxEF/ITxEF TAMS.aspx.

Sheng, Y., Miao, Z., Zhang, J., Lin, X., y Ma, H. (2018). Energy consumption model and energy benchmarks of five-star hotels in China. Energy and Buildings, 165, 286-292. DOI: 10.1016/j.enbuild.2018.01.031.

Tietenberg, T. y Lewis, L. (2012). Environmental \& Natural Resource Economics. 9th edition. New Jersey, United States of America: Pearson.

Tsoutsos, T., Tournaki, S., Frangou, M. y Tsitoura, M. (2017). Creating paradigms for nearly zero energy hotels in South Europe. AIMS Energy, 6(1): 1-18. DOI: 10.3934/energy.2018.1.1.

Wang, F., Lin, H. y Luo, J. (2017). Energy Consumption Analysis with a Weighted Energy Index for a Hotel Building. Procedia Engineering, 205 (2017): 1952-1958. DOI: 10.1016/j. proeng.2017.10.057

Williams, C. y Millington, A. (2004). The diverse and Contested Meanings of Sustainable Development. The Geographical Journal. Vol.170. No. 2, Environment and Development in the UK (Jun., 2004). Pp. 99-104.

Zabaloy, F., Recalde, M., \& Guzowski, C. (Mayo, 2017). Condiciones de entorno para la eficiencia energética: Las experiencias en el sector residencial de Latinoamérica. Trabajo presentado en VIII Congreso Nacional de Estudiantes de Posgrado en Economía (CNEPE) del Instituto de Investigaciones Económicas y Sociales del Sur, CONICET-UNS, Bahía Blanca, Argentina. Recuperado de https://www.iiess-conicet.gob.ar/images/actasCNEPE/ CNEPE8/zabaloy-recalde-guzowski.pdf. 


\section{Anexo 1 \\ ANEXO 1. ENCUESTA DE EFICIENCIA ENERGÉTICA EN HOTELES}

Instrumento para medir la eficiencia energética en empresas de alojamiento temporal en la zona conurbada del sur de Tamaulipas, México.

\section{CARACTERIZACIÓN SOCIOECONÓMICA DEL HOSPEDAJE}

1.- Nombre del establecimiento:

2.- Ubicación del hotel:

3.- De acuerdo con el sistema de clasificación hotelera, ¿Con qué categoría cuenta el establecimiento?
a) 1 estrella
b) 2 estrellas
c) 3 estrellas
d) 4 estrellas
e) 5 estrellas
f) Sin categoría

4.- ¿El establecimiento cuenta con la Constancia de Certificación Hotelera otorgada por la Secretaría de Turismo?
$\mathrm{Si}$ b) No
c) No sabe
d) Está en trámite
e) No contestó

5.- Número de habitaciones:

6.- Precio promedio por noche:

7.- ¿Cuántas personas laboran en el establecimiento?:

8.- ¿El hotel lleva un registro del consumo de energía eléctrica?
a) Diario
b) Mensual
c) Bimestral
d) Anual
e) No se lleva a cabo un registro

9.- ¿El establecimiento utiliza fuentes de energía renovable o eco certificadas?
a) Menos del $50 \%$
b) Entre $51 \%$ y $75 \%$
c) $76 \%$ al $100 \%$
d) No
e) No sabe

\section{CARACTERIZACIÓN ENERGÉTICA DEL HOSPEDAJE}

10.- ¿Qué fuente energética utiliza principalmente para el funcionamiento del hotel?
a) Gas de red (GN)
b) Electricidad
c) Tanque de Gas (LP)
d) Otros combustibles líquidos
e) Fuentes renovables de energía.

11.- ¿Qué fuentes energéticas utiliza para la cocción de alimentos?
a) Gas de Red (GN)
b) Electricidad
c) Tanque de Gas (LP)
d) Otros combustibles líquidos e) Fuentes de energía renovables

12.- ¿Qué fuentes energéticas utiliza para la refrigeración?
a) Gas de red (GN)
b) Electricidad
c) Tanque de Gas (LP)
d) Otros combustibles líquidos
e) Fuentes renovables de energía 
13.- ¿Qué fuentes energéticas utiliza para la iluminación?
a) Gas de red (GN)
b) Electricidad
c) Tanque de Gas (LP)
d) Otros combustibles líquidos

e) Fuentes renovables de energía

14.- ¿Qué fuentes energéticas utiliza para el calentamiento del agua?
a) Gas de red (GN)
b) Electricidad
c) Tanque de Gas (LP)
d) Otros combustibles líquidos

e) Fuentes renovables de energía

15.- ¿El establecimiento ha recibido visitas de inspección por parte del gobierno (local, estatal o federal) relacionadas con la eficiencia energética del hotel?
a) Local
b) Estatal
c) Federal
d) No
e) No sabe

\section{MANTENIMIENTO}

16.- ¿Se realizan cambios en el sistema de control para la calefacción o aire acondicionado de acuerdo a los cambios de clima por cuestión de temporadas de calor o frío?
a) Siempre
b) Frecuentemente
c) Algunas veces
d) Rara vez
e) No se realizan

17.- ¿Con qué frecuencia se realiza limpieza y/ o sustitución de los filtros de grasa en las campanas de cocina del establecimiento?
a) Una vez al mes
b) Una vez cada tres meses
c) Una vez al semestre
d) Una vez al año

18.- ¿Con qué frecuencia se realiza la limpieza de los extractores del sistema de ventilación?
a) Una vez al mes
b) Una vez cada tres meses
c) Una vez al semestre
d) Una vez al año

19.- ¿Con qué frecuencia se realiza la revisión del sistema de ventilación?
a) Una vez al mes
b) Una vez cada tres meses
c) Una vez al semestre
d) Una vez al año.

20.- ¿Cuántos eventos de reparación se llevaron a cabo en un año en el sistema de ventilación?
a) 1 a 3
b) 4 a 6
c) más de 7
c) No sabe
d) No contestó

21.- El hotel cuenta con una política formal (incluida en manuales de operación, descripción de actividades, reglamentos, políticas aprobadas, etc.) respecto a los mecanismos utilizados para el ahorro de energía.
a) $\mathrm{Si}$
b) No
c) No sabe
d) No contestó

22.- En temporada baja, el hotel mantiene cerrada una parte de las instalaciones (habitaciones, salones, áreas comunes, etc.) con la finalidad de ahorrar energía, como lo es: el aire acondicionado o calefacción, la iluminación, así como los aparatos que se encuentran dentro de las habitaciones.
a) $\mathrm{Si}$
b) No
c) No sabe
d) No contestó

23.- ¿Qué temperatura se establece como estándar para la calefacción y/o el aire acondicionado?
a) $22^{\circ}$
b) $27^{\circ}$
c) $18^{\circ}$
f) No se establece temperatura standard
g) No contestó 
24.- ¿Cuántas veces el establecimiento es impermeabilizado o aislado con la finalidad de reducir el consumo de energía?
a) Una vez cada año
b) Una vez cada 5 años
c) Una vez cada 10 años.
d) No se realiza

impermeabilizado

e) No sabe

25.- ¿La tubería de agua caliente del establecimiento se encuentra aislada correctamente?
a) $\mathrm{Si}$
b) No
c) No sabe
d) No contestó

26.- ¿Los aparatos como máquinas expendedoras de snacks, dispensadores de agua fría/ caliente o de café, se encuentran apagados durante períodos de baja ocupación o durante ciertas horas del día?
a) Durante la noche
b) Temporada de baja ocupación
c) Otro período
d) No se apagan e) No sabe

27.- ¿El establecimiento cuenta con políticas sobre lavado de sábanas en agua fría cuando sea posible y apropiado?
a) $\mathrm{Si}$
b) No
c) No sabe
d) No contestó

28.- ¿Qué tipo de iluminación se encuentra en las instalaciones del hotel? Mencione el porcentaje.
a) LED
b) Fluorescente
c) Halógeno
d) Incandescente
e) Otro

29.- ¿Las habitaciones incluyen minibares?
Todas
b) Algunas
c) Ninguna
d) No sabe

30.- ¿El establecimiento cuenta con un sistema de ahorro de energía en la iluminación exterior? Con sensores de movimiento o apagado controlado por temporizadores.
a) $\mathrm{Si}$
b) No
c) No sabe
d) No contestó

31.- ¿Cuenta el establecimiento con ventanas equipadas con algún tipo de aislante térmico?
a) Todas
b) Algunas
c) Ninguna
d) No sabe
e) No contestó.

32.- ¿Se encuentra restringida la apertura de ventanas en las habitaciones del establecimiento?
a) $\mathrm{Si}$
b) No
c) No sabe
d) No contestó

33.- En caso que la respuesta sea No, indique si el establecimiento cuenta con un sistema en las habitaciones que registre cuando una ventana se encuentra abierta y se procede a apagar el aire acondicionado o la calefacción de manera automática según sea el caso
a) $\mathrm{Si}$
b) $\mathrm{No}$
c) No sabe
d) No contestó

34.- ¿El establecimiento cuenta con aparatos eléctricos con certificados de eficiencia energética?
a) Menos del $50 \%$
b) Entre $51 \%$ y $75 \%$
c) $76 \%$ al $100 \%$
d) No
e) No sabe 
35.- ¿El establecimiento cuenta con calentadores con fuentes de calor directa como los son las resistencias eléctricas?
a) Menos del $50 \%$
b) Entre $51 \%$ y $75 \%$
c) $76 \%$ al $100 \%$
d) No
e) No sabe

36.- ¿El establecimiento cuenta con plantas de ventilación equipadas con ventiladores de optimización energética y motores de ahorro de energía?
a) $\mathrm{Si}$
b) No
c) No sabe
d) No contestó

37.- ¿El establecimiento cuenta con un sistema automático que apaga los aparatos eléctricos e iluminación al salir el huésped de la habitación?
a) Llave inteligente de tarjeta
b) Detectores de movimiento
c) Otro. Especifique
d) No
e) No sabe

38.- ¿El establecimiento cuenta con sensores automáticos para apagar la iluminación en salones y corredores que no se encuentren en uso?
a) Menos del $50 \%$
b) Entre $51 \%$ y $75 \%$
c) $76 \%$ al $100 \%$
d) No
e) No sabe

39.- ¿El establecimiento cuenta con sistemas automáticos de ahorro de energía en áreas comunes como son piscinas, gimnasios, restaurantes, bares u otros?
a) Menos del $50 \%$
b) Entre $51 \%$ y $75 \%$
c) $76 \%$ al $100 \%$
d) No
e) No sabe

40.- ¿El establecimiento está equipado con medidores de gas y energía eléctrica en lugares de alto consumo como cocina, gimnasios, calefacción en piscinas, aire acondicionado y calefactores centrales?
a) Menos del $50 \%$
b) Entre $51 \%$ y $75 \%$
c) $76 \%$ al $100 \%$
d) No
e) No sabe

41.- ¿El establecimiento cuenta con un sistema para la recuperación de calor utilizable en otra área?
a) Menos del $50 \%$
b) Entre $51 \%$ y $75 \%$
c) $76 \%$ al $100 \%$
d) $\mathrm{No}$
e) No sabe

42.- ¿Los aparatos electrónicos como computadoras, copiadoras, escáneres e impresoras cuentan con la característica denominado "modo de ahorro de energía o de apagado automático" después de cierto tiempo de inactividad?
a) $\mathrm{Si}$
b) No
c) No sabe
d) No contestó

43.- ¿El establecimiento utiliza campanas de cocina que cuentan con equipo automático de encendido cuando las parrillas se encuentran en uso o ante la presencia de vapor al cocinar?
a) $\mathrm{Si}$
b) No
c) No sabe
d) No contestó

44.- ¿Las tinas de hidromasaje, Sauna y Piscinas cuentan con temporizadores para el ahorro de energía?
a) $\mathrm{Si}$
b) No
c) No sabe
d) No contestó

45.- ¿Las tinas de hidromasaje y Piscinas están cubiertas durante el periodo en que no están en uso?
a) $\mathrm{Si}$
b) No
c) No sabe
d) No contestó 


\section{CONSTRUCCIÓN}

46.- ¿La construcción del inmueble tomó en cuenta el uso efectivo de la sombra para reducir costos de enfriamiento?
a) $\mathrm{Si}$
b) No
c) No sabe
d) No contestó

47.- ¿El establecimiento cuenta con instalación de muros o techos verdes?
a) $\mathrm{Si}$
b) No
c) No sabe
d) No contestó

Fecha de Aplicación 